- 11-18 year olds consume 8 times the recommended sugar allowance, which has not significantly decreased compared to 4-10 year olds.

- $46 \%$ of 15 year olds have decay in their permanent teeth

- $20 \%$ of secondary school aged children are already obese and trends show no improvement in obesity prevalence for children in Year 6 compared to children in reception.

- 5\% of 15 year olds smoke regularly

- 10-14 is peak age for diagnosis of Type 1 diabetes, with low rates of secondary complication screening

- Average age for child sexual exploitation concerns to be raised is $15-17$ years

Contrary to popular understanding, young people are frequent users of health services

- Half of Year 10 pupils report visiting their GP in the last 3 months.

- 369 out of 1000 young people aged 15-19 had an A\&E attendance in 2014/2015

Despite being frequent users of health services, services are not youth friendly

- Only a third of young people aged 18-24 report their GP appointment as good/very good.

- Training in adolescent health is not mandatory for GP practices

Conclusions The health of young people is not improving at the same rate as that of younger children, particularly in relation to obesity. These data provide a strong case for making young people's early intervention and age-appropriate health promotion a priority. It is vital that we collect data and commission services specially to meet the health needs of young people in order to prevent non-communicable diseases; caused by lifestyle behaviours that commonly start in adolescence.

\section{G423(P) FROM REFLECTION TO ACTION: A MULTIMODAL, INTERACTIVE APPROACH TO ENHANCING TRAINING ON CHILD REFUGEE HEALTH}

${ }^{1} \mathrm{G}$ Singh, ${ }^{2} \mathrm{C}$ Sen. ${ }^{1}$ Community Child Health, London North West Healthcare Trust, London, UK; ${ }^{2}$ Academic, Royal Society of Medicine, London, UK

\subsection{6/archdischild-2018-rcpch.412}

Aims Child refugees have specific but complex health needs that place significant demands on health systems and those working within them. Public and professional expectation is that health workers are proficient in caring for this vulnerable population. ${ }^{1-3}$ Despite increasing numbers of child refugees to the $\mathrm{UK},{ }^{4}$ health professionals report feeling ill-equipped to address these needs, and unable to fulfil their ethical obligations. $^{5}$

We designed a training course to address this gap, and evaluated the impact on professional confidence and competency in caring for child refugees.

Methods Our interdisciplinary, one-day course for child health professionals aimed to deepen understanding of: the geopolitical origins of the 'migration crisis'; the physical and mental health needs of child refugees; and advocacy for vulnerable populations. Following established pedagogical theory, ${ }^{6}$ we utilised a multimodal and interactive model: digital technology; skills workshops; first-hand testimonies and the use of literary and visual art were employed alongside more traditional lectures. An online pre- and post-course questionnaire with multiple-choice and free-text questions captured professionals' perceptions about confidence and competence.

Results Of the 190 course delegates, responses were as follows (table 1):

\begin{tabular}{lll} 
Abstract G423(P) Table 1 & & \\
\hline 'I feel confident to deal with child refugee...' & Pre-course & Post-course \\
\hline - Physical needs & $34 \%(31 / 91)$ & $88 \%(60 / 88)$ \\
- Mental health needs & $9 \%(8 / 88)$ & $43 \%(32 / 74)$ \\
\hline
\end{tabular}

For delegates, the biggest obstacles to managing the 'migrant crisis' were: lack of awareness of problems faced by refugees $(47 \%$ - 50/107) and lack of collaboration between health and social care $(31 \%$ - 33/107). Free-text responses consistently reflected a desire to learn how to: advocate for child refugees in both local and national spheres; educate other professionals and the public about refugee needs; and be more active politically and as a volunteer.

Conclusion This study confirms significant demand for professional training around child refugee health needs. Our interdisciplinary, multimodal and interactive approach increased confidence of delegates to manage child refugee physical and mental health, stimulated interagency collaboration and provided a platform for actualising professional ethical and advocacy responsibilities to vulnerable populations. This model has potential for professional training elsewhere.

\section{G424(P) THE IMPACT OF AGE AND DEDICATED AGE- APPROPRIATE SERVICES ON TRANSITION OUTCOMES FROM PAEDIATRIC TO ADULT HEALTH SYSTEMS: A REVIEW OF REVIEWS}

AA Yassaee, D Hale, A Armitage, R Viner. General Adolescent Paediatrics Unit, Institute of Child Health, University College London, London, UK

\subsection{6/archdischild-2018-rcpch.413}

Aims To identify healthcare transition models associated with positive outcomes, and review evidence regarding the optimum age of transfer and the value of existing age-appropriate services.

Methods A systematic search strategy was used across multiple databases to identify systematic reviews addressing two review questions.

- What is the evidence for a delayed transition?

- What is the value of existing age-appropriate services?

Reviews that were not systematic, did not cover healthcare transitions, did not concern long-term conditions, or were not reviews of primary literature were excluded. Reviews of quantitative, qualitative or mixed methods studies were included. Quality was assessed using the R-AMSTAR tool. Studies with a score below 22 were excluded. The search strategy was initially applied in April 2015 and was repeated in May 2017 to include newly published literature.

Results The initial search strategy produced 2533 results with 11 systematic reviews meeting all inclusion criteria. Repeating the search criteria produced 3616 new results published between April 2015 and May 2017, with an additional 13 